\title{
Difference in Pulse Arrival Time at Forehead and at Finger as a Surrogate of Pulse Transit Time
}

\author{
Jesús Lázaro ${ }^{1,2}$, Raquel Bailón ${ }^{1,2}$, Pablo Laguna ${ }^{1,2}$, \\ Vaidotas Marozas $^{3}$, Andrius Rapalis ${ }^{3}$, Eduardo Gil ${ }^{1,2}$ \\ ${ }^{1}$ BSICoS Group, Aragón Institute for Engineering Research, IIS Aragón, University of Zaragoza, \\ Zaragoza, Spain \\ ${ }^{2}$ CIBER in Bioengineering, Biomaterials and Nanomedicine (CIBER-BBN), Madrid, Spain \\ ${ }^{3}$ Biomedical Engineering Institute, Kaunas University of Technology, Kaunas, Lithuania
}

\begin{abstract}
Pulse transit time (PTT) difference (PTTD) to the forehead and finger dynamics are compared to pulse arrival time towards the finger (PATF) dynamics during a tilt table test. Two frequency bands, where different physiological information is expected, are analyzed: low frequency (LF) influenced by both sympathetic and parasympathetic activity, and high frequency $(H F)$ influenced by parasympathetic activity. As PATF, PTTD is influenced by PTT, but in contrast to PATF, PTTD is not influenced by the preejection period (PEP). This is advantaging in certain applications such as arterial stiffness assessment or blood pressure estimation.

Results showed higher correlation between PTTD and PATF during rest stages than during tilt stage, when the PEP dynamics have stronger effect in PATF dynamics. This suggests that PTTD variability can potentially be a surrogate of PTT variability that is not influenced by PEP, which is advantaging in the previously mentioned applications. However, further studies must be elaborated in order to evaluate the potential of PTTD in such specific applications.
\end{abstract}

\section{Introduction}

Pulse transit time (PTT) is defined as the time that the pressure wave takes to propagate between two points of the arterial branches. PTT represents a tool for assessing arterial stiffness, and it also seems to be the best basis for a non-invasive-ubiquitous-cuff-less BP monitor [1].

Two points of the arterial tree are needed for the PTT computation: the proximal and the distal points. The last one, distal point, is usually measured at the periphery based on pulse photoplethysmographic (PPG) signal. The first one, proximal point, is usually the aortic valve. As the instant when the aortic valve opens is very difficult to be accurately detected, a surrogate is usually used. Probably, the most used surrogate is the R-wave in the electrocardiogram (ECG), leading to the so-called pulse arrival time (PAT). PAT is not exactly the PTT, as it includes the time interval between ventricular depolarization and the opening of the aortic valve which is known as pre-ejection period (PEP) and it varies beat-to-beat. These variations make the PAT more inconvenient than the PTT for certain applications, such as arterial stiffness assessment [2] or blood pressure (BP) estimation, specially when estimating diastolic and/or mean BP [1].

This motivates the study of some alternatives to ECG for measuring the proximal point, including ballistocardiogram, seismocardiogram, and impedance cardiography [1]. Another approach to avoid the influence of PEP consists of measuring the difference of 2 distal instants, getting the difference of two PATs affected by the same PEP and so, PTT difference (PTTD). As PTT, PTTD has been studied for arterial stiffness assessment [3], and it also has been studied as a surrogate of PTT [4]. However, the study in [4] reported an analysis of correlation of PTTD and PAT signals during rest conditions within the whole spectrum, while several modulations with different physiological origins are expected in PTT signals $[5,6]$. In this paper, an analysis of the correlation between PTTD and PAT towards the finger (PATF) dynamics in different frequency bands during a tilt table test is presented.

\section{Materials and methods}

\subsection{Data and preprocessing}

ECG and PPG data were simultaneously recorded from 14 volunteers (10 male). The protocol consisted of $10 \mathrm{~min}$ utes in early supine position (Rest1), followed by 5 minutes tilted up $80^{\circ}$ (Tilt), and 5 minutes back to supine position (Rest2). The automatically controlled tilt table takes 
1 minute to change position.

Specifically, these data included ECG lead II, a transmission PPG signal at the index finger and a reflection PPG signal acquired at the forehead, all of them acquired by Cardioholter6.2-8E78 (BMII, Lithuania). The sampling rate was $500 \mathrm{~Hz}$ for the ECG signal and $250 \mathrm{~Hz}$ for both PPG signals. Cubic spline interpolation was used for obtaining a $1000 \mathrm{~Hz}$ version of each signal in order to increase the time resolution of the fiducial points.

For the 3 signals, the baseline was attenuated by applying a high-pass filter with a cut-off frequency of $0.3 \mathrm{~Hz}$. In addition, a low-pass filter was applied to the PPG signals using a cut-off frequency of $35 \mathrm{~Hz}$ in case of the transmission PPG signal, and $15 \mathrm{~Hz}$ in case of the reflection signal which is lower due to the smoother variations of this signal. Artifacts in PPG signals were automatically detected by an algorithm based on Hjorth parameters [7] and corrected by visual inspection.

\subsection{Estimation of parameters}

The maximum of the $\mathrm{R}$ wave of each QRS complex $\left(n_{\mathrm{R}_{i}}\right)$ was detected by using a wavelet-based algorithm [8]. The fiducial point chosen for the PPG signals was the medium-amplitude point $\left(n_{\mathrm{MF} i}\right.$ and $\left.n_{\mathrm{MH} i}\right)$, for the fingerand the head-PPG signals, respectively), defined as that instant when the PPG pulse have reached the half of its amplitude. These points were automatically detected by using a low-pass-derivative-filter-based algorithm [9].

PAT was measured as the difference of those fiducial points detected at the finger-PPG signal and those detected in the ECG signal:

$$
x_{\mathrm{PATF}}^{u}\left(n_{\mathrm{MF} i}\right)=\left[n_{\mathrm{MF} i}-n_{\mathrm{R}_{i}}\right] \delta\left(n_{\mathrm{MF}_{F}}\right),
$$

where the superscript " $u$ " denotes that the signal is unevenly sampled. Similarly, $x_{\mathrm{PATH}}^{u}\left(n_{\mathrm{MF} i}\right)$ was computed as the difference of $n_{\mathrm{MH} i}$ and $n_{\mathrm{R}_{i}}$.

As the finger PAT and forehead PAT are affected by the same PEP, the PTTD can be measured as the PAT difference, i.e.:

$$
x_{\mathrm{PTTD}}^{u}\left(n_{\mathrm{MF}_{\mathrm{F} i}}\right)=\left[n_{\mathrm{M}_{\mathrm{F} i}}-n_{\mathrm{M}_{\mathrm{H} i}}\right] \delta\left(n_{\mathrm{MF} i}\right) .
$$

Subsequently, $4-\mathrm{Hz}$ evenly sampled versions of $x_{\mathrm{PATF}}^{u}\left(n_{\mathrm{MF} i}\right), x_{\mathrm{PATH}}^{u}\left(n_{\mathrm{MF} i}\right)$, and $x_{\mathrm{PTTD}}^{u}\left(n_{\mathrm{MF} i}\right)$ were obtained by cubic spline interpolation, being denoted $x_{\mathrm{PATF}}(n), x_{\mathrm{PATH}}(n)$, and $x_{\text {РтTD }}(n)$, respectively. An example of these signals is shown in Fig. 2. Definitions of PATF, PATH, and PTTD are illustrated in Fig. 1.

\subsection{Correlation and power analyses}

Two different 300-order-FIR-band-pass filters were applied to $x_{\mathrm{PATF}}(n), x_{\text {РАTH }}(n)$, and $x_{\mathrm{PTTD}}(n)$ signals. These band-pass filters correspond to two of the classical bands for heart rate variability (HRV) analysis [10], as physiological information is expected in these two bands in PTT:

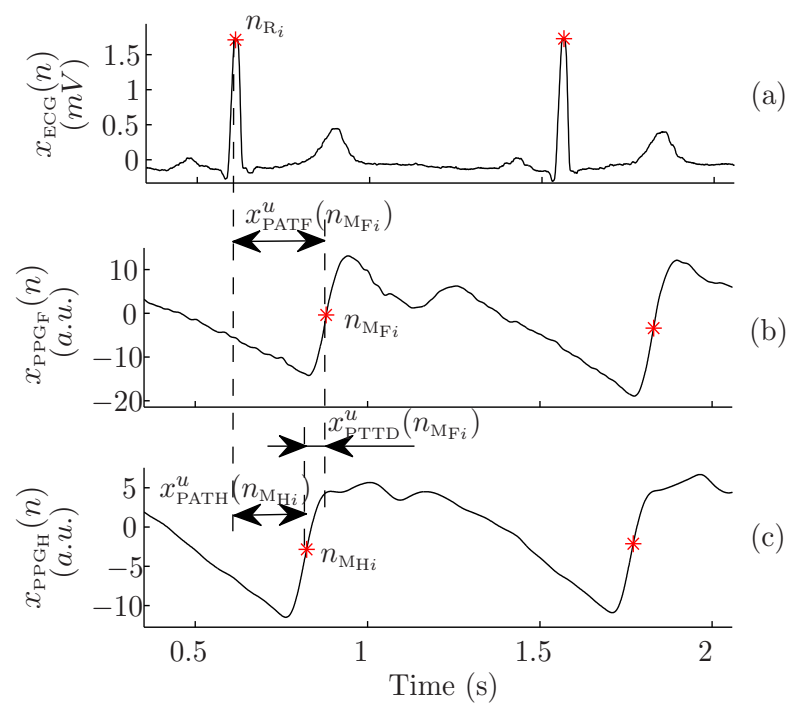

Figure 1. Example of ECG (a), and finger (b) and forehead PPG (c) signals, and definitions of PATF, PATH, and PTTD.

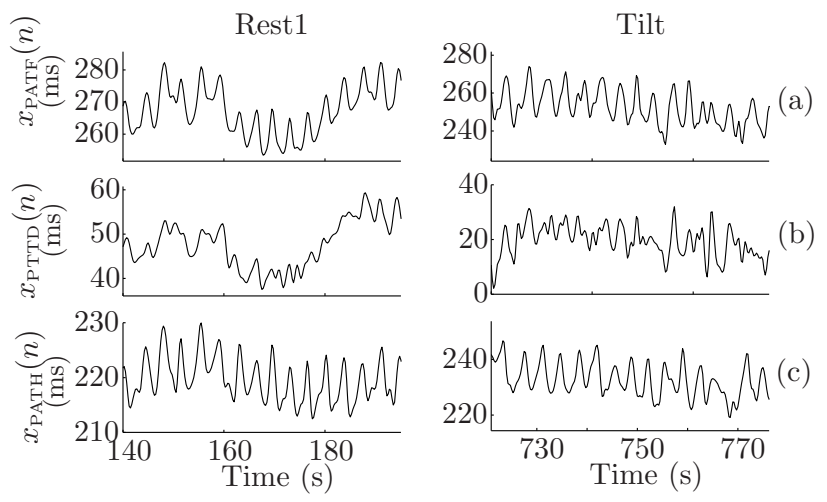

Figure 2. Example of PATF (a), PTTD (b), and PATH (c) signals during Rest 1 and Tilt.

- Low frequency (LF): $[0.04,0.15] \mathrm{Hz}$. As PTT is related to BP, PTT is expected to follow the Mayer wave (sympathetic-associated) [5] which is typically within this band.

- High frequency (HF): $[0.15,0.4] \mathrm{Hz}$. PTT is expected to follow the respiration (parasympathetic-associated) [6], which is typically within this band.

The resulting band-pass signals were analyzed in $30 \mathrm{~s}$ length running windows with a $50 \%$ of overlap for subsequent analysis. Correlation coefficient between each pair of signals were computed for both bands. Before computing the correlation coefficient within each time window and frequency band, the delay between two signals was estimated (and corrected) as the lag which maximizes their cross correlation (accepting a maximum delay of $1 \mathrm{~s}$ ).

In addition, the power within $\operatorname{LF}\left(P_{\mathrm{LF}}\right)$ and $\operatorname{HF}\left(P_{\mathrm{HF}}\right)$ were computed for the same time windows by using $x_{\mathrm{P} \text { РтD }}(n)$, 


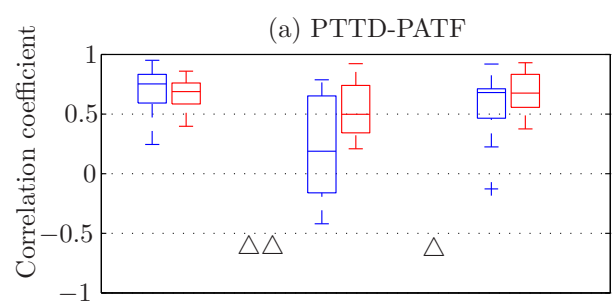

(d) PTTD

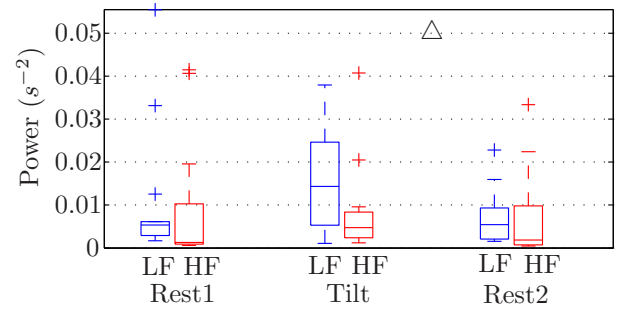

(b) PATF-PATH

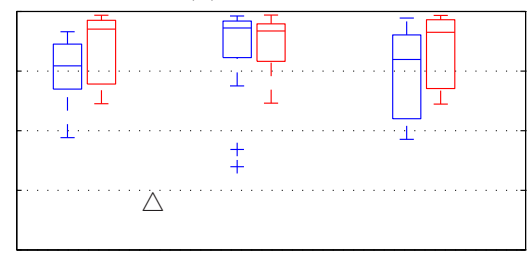

(e) PATF

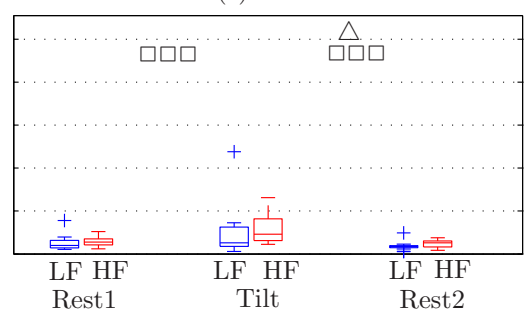

(c) PTTD-PATH

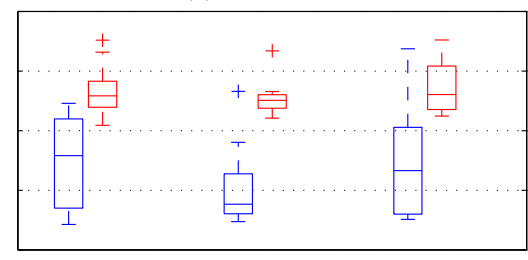

(f) PATH

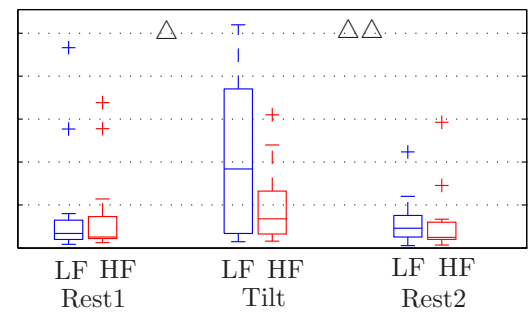

Figure 3. Boxplots of obtained intra-subject medians of obtained correlation coefficients between PTTD-PATF (a), PATFPATH (b), and PTTD-PATH (c), and boxplots of obtained intra-subject medians of $P_{\mathrm{LF}}$ and $P_{\mathrm{HF}}$ of PTTD (d), PATF (e), and PATH (f), for each frequency band and stage of the protocol. Significant differences according to the Wilcoxon paired statistical test are denoted between compared groups with $\triangle$ for LF band ( $\triangle$ if $p<0.05, \triangle \triangle$ if $p<0.01$, and $\triangle \triangle \triangle$ if $p<0.001$ ) and, similarly, with $\square$ for HF band.

$x_{\mathrm{PATF}}(n)$, and $x_{\mathrm{PATH}}(n)$ and the classical periodogram.

\section{Results}

Figure 3 illustrates the obtained intra-subject medians of correlation coefficients between PTTD-PATF, PATFPATH, and PTTD-PATH. Obtained intra-subject medians of $P_{\mathrm{LF}}$ and $P_{\mathrm{HF}}$ of PTTD, PATF, and PATH, for each stage of the protocol are also illustrated. Significant differences $(p<0.05)$ according to the Wilcoxon paired statistical test are also shown in Fig 3. Note that no significant differences were found between Rest 1 and Rest 2 in any case.

\section{Discussion and conclusions}

This paper presents an analysis of correlation between the PTTD and PATF dynamics in different frequency bands. Dividing the analysis in frequency bands is important because several modulations with different physiological origins are expected in PTT signals and, therefore, a PTT signal can follow these different modulations in a different manner. For a better understanding, correlations between PATF and PATH, and between PTTD and PATH have been also analyzed.

The proportionality of PTTD and PATF/PATH is not in the same direction along the time. A possible explanation to this observation could be different actions of the autonomic nervous system in blood vessels from the heart to the finger than to the forehead. For example, a sympathetic activation stiffs arteries making faster both pressure waves, towards the finger (PWF) and towards the forehead
(PWH). The effect in $x_{\mathrm{PATF}}(n)$ and $x_{\mathrm{PATH}}(n)$ is a decrease, while the effect in $x_{\text {PTTD }}(n)$ depends on which pressure wave is more accelerated: the effect in $x_{\text {PTTD }}(n)$ is also a decrease if PWF is more accelerated than PWH, but the effect is an increase if PWH is the one more accelerated. Another possible effect is the difference of lengths of the paths traveled by PWF and PWH. The effect of a pressure wave velocity change in its associated PAT depends on the traveled space: the more traveled space, the more effect in PAT of a velocity change.

Powers of $x_{\text {PTtD }}(n), x_{\mathrm{PATF}}(n)$, and $x_{\mathrm{PATH}}(n)$ within LF and $\mathrm{HF}$ bands were also studied. Obtained $P_{\mathrm{HF}}$ for $x_{\mathrm{PATF}}(n)$ was significantly higher during Tilt $\left(4.59 \mathrm{E}-3 \mathrm{~s}^{2}\right)$ than during rest stages $\left(2.77 \mathrm{E}-3\right.$ and $\left.2.58 \mathrm{E}-3 \mathrm{~s}^{2}\right)$ according to the Wilcoxon paired test, while significant differences for $P_{\mathrm{LF}}$ were found only when comparing Tilt $\left(2.61 \mathrm{E}-3 \mathrm{~s}^{2}\right)$ to Rest2 $\left(1.74 \mathrm{E}-3 \mathrm{~s}^{2}\right)$. It is worthy to note that more data are available for Rest 1 (10 minutes) than for Rest2 (5 minutes). On the other hand, $P_{\mathrm{LF}}$ of $x_{\mathrm{PATH}}(n)$ was significantly higher during Tilt $\left(18.38 \mathrm{E}-3 \mathrm{~s}^{2}\right)$ than during rest stages (6.50E-3 and 7.58E-3 $\mathrm{s}^{2}$ ). These observations suggest that respiration effect in $x_{\mathrm{PATF}}(n)$ is stronger during Tilt, and they suggest also that $x_{\mathrm{PATH}}(n)$ is more affected by the sympathetic tone than $x_{\mathrm{PATF}}(n)$.

PTTD and PAT from 12 subjects were analyzed during stationary conditions in [4]. A correlation coefficient was computed for each subject, from the entire PTTD and PATF signals and for the whole frequency spectrum. The inter-subject mean \pm standard deviation were reported, and they vary from $0.05 \pm 0.18$ to $0.86 \pm 0.06$ depending on the used fiducial points. As the signals were analyzed 
in the whole spectrum, the computed correlations may be much more affected by one of the modulations being studied in this paper, or even by lower frequency components such as signal trends.

Results obtained in this study show a high correlation between PTTD and PATF in LF band (0.75/0.24, median/interquartile range) and a little lower for $\mathrm{HF}$ $(0.69 / 0.18)$ in Rest1. No significant differences were found with the values obtained for Rest $2(0.68 / 0.25$ and $0.68 / 0.28$, respectively) according to the Wilcoxon paired test.

A correlation coefficient of 0.85 between PAT towards the toe and PTTD to the toe and finger was reported in [3], where an analysis during a less aggressive tilt test $\left(30^{\circ}\right)$ was presented. In this study, the tilt stage is more aggressive $\left(80^{\circ}\right)$ and obtained correlation during this stage is lower $(0.19 / 0.81$ for LF and $0.50 / 0.40$ for HF), and it is also signifcantly lower than those obtained during rest stages according to the Wilcoxon paired test for LF. The reason of this observation may be that PEP dynamics have a stronger effect in PATF dynamics during tilt stage. It is worthy to note that PATF and PATH obtained a higher correlation within LF during tilt stage $(0.86 / 0.31)$ than for rest stages $(0.54 / 0.38$ and $0.60 / 0.70)$, being significantly different in the case of Rest1. This observation is coherent with the hypothesis of that PEP dynamics have a stronger effect in PAT dynamics during tilt stage. This hypothesis is also coherent with [11] where correlation between PEP and PATF is reported to be higher as the tilt angle increases. This PEP dynamics predominance in PAT dynamics may be due to the increase of sympatho-vagal balance induced by tilt, or it may be due to the postural change. One limitation of this study is the impossibility of separating both effects.

Another limitation of this study is the absence of a respiratory signal which would allow us to ensure that respiration is in HF band, and/or removing those subjects whose respiration is outside HF band in order not to confound the physiological interpretation of the LF and HF bands.

In conclusion, results suggest that PTTD is a noninvasisve measure potentially correlated with PTT and it is not influenced by PEP, which is advantaging in some applications such as arterial stiffness assessment or BP estimation. PTTD to the Forehead and Finger can be measured by two photoplethysmographs, making it suitable for implementation in wearable devices and smartphones [4]. However, further studies must be elaborated in order to evaluate the potential of PTTD in such specific applications.

\section{Acknowledgements}

This work was supported by Ministerio de Economía y Competitividad (MINECO), FEDER; under projects TIN2014-53567-R, TIN2013-42140-R, TEC2013-42140-
$\mathrm{R}$ and FIS-PI12/00514, by CIBER in Bioengineering, Biomaterials Nanomedicine (CIBER-BBN) through Instituto de Salud Carlos III, and by Grupo Consolidado BSICoS (T96) from DGA (Aragón) and European Social Fund (EU).

\section{References}

[1] Mukkamala R, Hahn JO, Inan OT, Mestha LK, Kim CS, Töreyin H, Kyal S. Toward ubiquitous blood pressure monitoring via pulse transit time: theory and practice. IEEE Trans Biomed Eng 2015;62:1879-1901.

[2] Payne RA, N SC, Webb DJ, Maxwell SRJ. Pulse transit time measured from the ECG: an unreliable marker of beatto-beat blood pressure. J Appl Physiol 2005;100:136-141.

[3] Nitzan M, Khanokh B, Slovik Y. The difference in pulse transit time to the toe and finger measured by photoplethysmography. Phys Meas 2002;23:85-93.

[4] Liu H, Ivanov K, Wang Y, Wang L. Toward a smartphone application for estimation of pulse transit time. Sensors 2015;15:27303-27321.

[5] Julien C. The enigma of Mayer waves: Facts and models. Cardiovascular Research 2006;70:12-21.

[6] Chua CP, Heneghan C. Pulse transit time-derived respiratory parameters and their variability across sleep stages. In Engineering in Medicine and Biology Society, 2005. Proceedings of the 27th Annual International Conference of the IEEE, volume 6. 2005; 6153-6156.

[7] Gil E, Vergara JM, Laguna P. Detection of decreases in the amplitude fluctuation of pulse photoplethysmography signal as indication of obstructive sleep apnea syndrome in children. Biomed Signal Process Control 2008;3(3):267277.

[8] Martínez JP, Almeida R, Olmos S, Rocha AP, Laguna P. A wavelet-based ECG delineator: Evaluation on standard databases. IEEE Trans Biomed Eng 2004;51:570-581.

[9] Lázaro J, Gil E, Vergara JM, Laguna P. Pulse rate variability analysis for discrimination of sleep-apnea-related decreases in the amplitude fluctuations of pulse photoplethysmographic signal in children. IEEE J Biomed Health Inform 2014;18:240-246.

[10] Task Force of the European Society of Cardiology and the North American Society of Pacing and Electrophysiology. Heart rate variability: standards of measurement, physiological interpretation and clinical use. Circulation 1996; 93(5):1043-1065.

[11] Chan GSH, Middleton PM, Celler BG, Wang L, Lovell NH. Change in pulse transit time and pre-ejection period during head-up tilt-induced progressive central hypovolaemia. J Clin Monit Comput 2006;70:12-21.

Address for correspondence:

Jesús Lázaro

Dep. Ingeniería Electrónica y Comunicaciones. Universidad de Zaragoza, C/ María de Luna 1, 50018 Zaragoza, Spain jlazarop@unizar.es 